\section{Case Reports in Neurology}

\title{
Dissociated Crossed Speech Areas in a Tumour Patient
}

\author{
Jörg Mauler ${ }^{a}$ Irene Neuner ${ }^{a, b} \quad$ Georg Neuloh $^{c}$ Bruno Fimm ${ }^{d}$ \\ Frank Boers $^{\mathrm{a}}$ Martin Wiesmann $^{\mathrm{e}}$ Hans Clusmann $^{\mathrm{c}}$ \\ Karl-Josef Langen ${ }^{a, f, g} \quad$ N. Jon Shaha, d, f \\ ${ }^{a}$ Institute of Neuroscience and Medicine-4, Forschungszentrum Jülich, Jülich, Germany; \\ ${ }^{b}$ Department of Psychiatry, Psychotherapy and Psychosomatics, RWTH Aachen University, \\ Aachen, Germany; ' Department of Neurosurgery, RWTH Aachen University, \\ Aachen, Germany; ${ }^{d}$ Department of Neurology, RWTH Aachen University, \\ Aachen, Germany; ${ }^{\mathrm{e}}$ Department of Neuroradiology, RWTH Aachen University, \\ Aachen, Germany; ${ }^{f}$ Jülich Aachen Research Alliance (JARA) - Translational Brain Medicine, \\ Aachen and Jülich, Germany; ${ }^{9}$ Department of Nuclear Medicine, RWTH Aachen University, \\ Aachen, Germany
}

\section{Keywords}

Dissociated crossed speech areas - Brain tumour - Functional magnetic resonance imaging · Wada test · Cortico-subcortical stimulation mapping · Tumour-induced brain plasticity · Case report

\begin{abstract}
In the past, the eloquent areas could be deliberately localised by the invasive Wada test. The very rare cases of dissociated crossed speech areas were accidentally found based on the clinical symptomatology. Today functional magnetic resonance imaging (fMRI)-based imaging can be employed to non-invasively localise the eloquent areas in brain tumour patients for therapy planning. A 41-year-old, left-handed man with a low-grade glioma in the left frontal operculum extending to the insular cortex, tension headaches, and anomic aphasia over 5 months underwent a pre-operative speech area localisation fMRI measurement, which revealed the evidence of the transhemispheric disposition, where the dominant Wernicke speech area is located on the left and the Broca's area is strongly lateralised to the right hemisphere. The outcome of the Wada test and the intraoperative cortico-subcortical stimulation mapping were congruent with this finding. After tumour removal, language area func-
\end{abstract}


tion was fully preserved. Upon the occurrence of brain tumours with a risk of impaired speech function, the rare dissociate crossed speech areas disposition may gain a clinically relevant meaning by allowing for more extended tumour removal. Hence, for its identification, diagnostics which take into account both brain hemispheres, such as fMRI, are recommended.

\section{Introduction}

Previously, among reports of anomalies of cerebral organisation such as crossed nonaphasia [1], rare cases of particularly dissociated crossed speech areas were reported when the transhemispheric configuration of speech areas had accidently been found based on the clinical symptomatology following vascular diseases, such as strokes [2]. When patterns of cerebral organisation were examined by internal intra-carotid amobarbital injection, the characteristics of bilaterality referred to the function of hemispheres in total [3]. Nowadays, modern functional magnetic resonance imaging (fMRI)-based techniques non-invasively facilitate 3D spatially resolved language network mapping. In patients with brain tumours located close to the eloquent areas, the transhemispheric pattern may have a significant impact on possible therapy options. We report on a man with an astrocytoma WHO II in the left frontal operculum.

\section{Case Report}

After suffering from tension headaches for 5 months, as well as right-side tinnitus, vertigo, increasing memory deficits, and anomic aphasia, a 41-year-old, left-handed for 9 out of 10 uni-manual activities (Edinburgh handedness inventory [4]) male clerk underwent neuropsychological assessment, diagnostic cranial MRI, CT, and PET using 0-(2-[18F]fluorethyl)L-tyrosine (FET) indicating a low-grade glioma in the left frontal operculum extending to the insular cortex. He had no evidence of an early-life structural lesion.

A comprehensive neuropsychological test battery was administered prior to operation, consisting of computerized and paper-pencil tasks. The Regensburg Word Fluency Test showed impaired performance on letter fluency and alternating category fluency. Furthermore, the patient showed average performance in vocabulary, verbal reasoning, spatial sense, figure-ground segmentation, and processing of anagrams with no significant differences between verbal and nonverbal subtests but with visuo-constructive abilities slightly above average. There was an overall average performance in verbal and spatial learning and short-term memory except below-average performance (percentile $<5$ ) in delayed recall in a German variant of the Rey Auditory Verbal Learning Test. The Test of Attentional Performance showed unimpaired alertness and normal selective visual/divided attention but slightly reduced flexibility of attention.

Surgical tumour removal and adjuvant therapy was planned. The eloquent areas were localized using an fMRI-language network mapping paradigm [5] $(4 \times 36 \mathrm{~s}$ stimulation periods nested into 5 resting periods of $18 \mathrm{~s}$ duration each) comprising word generation, where the patient had to find examples matching generic terms presented on a display, and sentence generation where pictures had to be transferred into sentences. Before the measurement, the patient was trained to fluently generate speech. Both paradigms ran twice with patient confirmation each time that he generated language fluently. In order to minimise 
motion artefacts, the patient's head was immobilised with cushions and the patient was asked just to whisper instead of speaking in a loud voice. The data was acquired at 3T with an 8-channel PA head coil. Functional scans were carried through with an EPI sequence $(\mathrm{TR}=3 \mathrm{~s}$, matrix size $64 \times 64$, in-plane resolution $3 \mathrm{~mm}, 32$ slices in ascending order, slice thickness $3 \mathrm{~mm}$, inter-slice gap $1.2 \mathrm{~mm}$ ). The analysis was performed under SPM 8 (realignment/re-slicing, co-registration to the MPRAGE data set, $5 \mathrm{~mm}$ kernel smoothing [isotropic, Gaussian], fit of the general linear model, contrast settings [speech generation vs. baseline], and presentation with the family-wise error [FEW] $p=0.05$ /cluster threshold 0 voxel).

As the combined analysis of fMRI language paradigms enhances the assessment of hemispheric dominance, the results of the word and sentence generation tasks were evaluated in conjunction [6]. Strong visual cortex activation served as a positive measurement control. Both runs of the word and sentence generation paradigms yielded two spots of strong signal intensity in the left dorsal superior temporal gyrus/sulcus, remote from the tumour in the left frontal region (Fig. 1a). At the corresponding position on the contralateral side, a small activation cluster was located, classifiable as the homologue of the more prominent Wernicke speech area (following the classical terminology, which eases the anatomical characterisation of the activated areas) residing on the left.

Only the first run of the word generation paradigm suggested activation in the grey matter of the left inferior frontal gyrus (Fig. 1b). A clear activation appeared in the right frontal lobe, comprising activation clusters of the first run of the word generation and of both runs of the sentence generation paradigm (Fig. 1c).

Although the validity of fMRI-based language localisation has been shown in comparison to the Wada test and intraoperative cortical mapping on tumour patients [7], the results were not perfectly congruent. Other publications even showed tumour-induced neurovascular uncoupling causing contralateral pseudo-dominance at fMRI in few cases [8]. In order to validate the conspicuous fMRI findings, which revealed the transhemispheric configuration (dominant Wernicke area: left, Broca centre: strongly lateralized to the right hemisphere), the Wada test [9] was carried out. A 5F guide catheter (5F Envoy, Codman, Raynham, MA, USA) was advanced into the cervical part of the left internal carotid artery (ICA). Angiography confirmed that both anterior and posterior communicating arteries were present, but no cross-flow occurred. First, 120 mg amobarbital (Amytal Sodium, Marathon Pharmaceuticals, Northbrook, IL, USA) was injected into the left ICA. Neuropsychological testing confirmed complete right-sided hemiparesis and severe disturbance of speech. Following motor and speech function recovery, a microcatheter (Echelon 14, Covidien, Dublin, Ireland) was advanced into the anterior M2 branch of the left middle cerebral artery (MCA). This branch supplied only the anterior part of the left MCA territory including frontal operculum and insula. Sixty minutes after the first injection, $40 \mathrm{mg}$ amobarbital were injected through the microcatheter and neuropsychological testing was repeated.

The Wada test led to a manifest disturbance of speech after the left ICA amobarbital injection, but just to a mild disturbance when only the anterior part of the left MCA territory, including the frontal operculum and the insula, were selectively anaesthetized. Surgery was performed under analgo-sedation in order to perform cortico-subcortical stimulation mapping for language function and continuous language testing in the conscious patient during image-guided tumour removal. Cortico-subcortical language mapping was performed by inhibitory electrical brain stimulation with the patient performing a simple picture-naming paradigm [10]. Stimulation intensity at $3 \mathrm{~mA}$ was chosen below the 4-mA threshold for afterdischarges in the electrocorticogram. During tumour resection, the patient continued to perform the naming test for continuous functional monitoring. 
Intraoperative stimulation mapping did not yield language functions (naming) within and adjacent to the operative field. Resection was abandoned when continuous language testing yielded perseverations when resecting the deepest part of the tumour. Postoperative MRI revealed minor tumour remnants deep in the resection cavity.

Language area function was fully preserved except for a mild anomia which had resolved completely at discharge. Histological findings revealed a diffuse astrocytoma WHO II.

\section{Discussion}

For the sake of conciseness, the activated brain areas are described in terms of the classical Broca and Wernicke areas, but more realistically, these regions should rather be seen as important nodes of a larger language network than as phrenological locations. Irrespective of the referred model, these regions must not be impaired in surgical interventions.

In contrast to the activation cluster in the left frontal lobe, right frontal lobe activity was significantly stronger, giving distinct evidence of the Broca's area [11] being lateralised to this very side. In case of brain tumours, which generally may cause vascular compression or implicate neovascularisation, the resulting localisation and the activated volume may be altered [12]. Moreover, fMRI data provides only qualitative information such as position and relative level of activation. The size of the activation clusters depends on statistical threshold effects and so does not represent the absolute spatial extent of the underlying functional area [13]. In spite of these limitations, the fMRI-based finding of the Broca's area being lateralised to the right side is supported by the Wada test outcome, which revealed only a minor reduction of speech after the selective anaesthesia of the left frontal operculum. Moreover, the intraoperative language mapping result was consistent with this interpretation. Nevertheless, the potential occurrence of bilaterality in terms of language function representation in both hemispheres must be addressed. The information of at least one of the invasive methods applied to the contralateral hemisphere would have been desirable, but with the methods of clinical confirmation described here, these measurements were not admissible due to ethical reasons.

The pattern of strong activation in the left temporal lobe and of minor activation on the contralateral side supports the finding of the Wernicke speech area being lateralised to the left side, in agreement with the Wada test result. Barbiturate injection into the left MCA caused a major disturbance of speech, whereas selective deactivation of the frontal vascular territory induced only marginal speech deprivation.

The rare transhemispheric pattern, Broca's area located on the right, Wernicke's speech area lateralised to the left, allowed for less risky surgery in the area of the left inferior frontal gyrus with respect to language function integrity, which is subject to limitations owing to the undetermined spatial extent of this area due to its threshold dependency [13]. The configuration may show a case of tumour-induced brain plasticity $[8,14,15]$, but due to missing fMRI/clinical data from the time when the patient was healthy, this hypothesis cannot be proven.

In conclusion, this report shows a case where the dissociated crossed speech area configuration has a clinical impact on surgical treatment planning, where fMRI-based language localisation - usually regarded as a method for research - substantially contributes to this finding in a clinical application. It is recommended that such examinations be performed in comparable cases. 


\section{Acknowledgments}

The authors wish to thank Dr. Gabriele Stoffels for the acquisition of the anatomical MRI and FET uptake data.

\section{Statement of Ethics}

The study was approved by the local ethics committee and federal authorities. The patient gave written informed consent for his participation in the study and publication.

\section{Disclosure Statement}

The authors declare no conflict of interest.

\section{References}

1 Alexander MP, Annett M: Crossed aphasia and related anomalies of cerebral organization: case reports and a genetic hypothesis. Brain Lang 1996;55:213-239.

2 Paparounas K, Eftaxias D, Akritidis N: Dissociated crossed aphasia: a challenging language representation disorder. Neurology 2002;59:441-442.

3 Kurthen M, Helmstaedter C, Linke DB, Hufnagel A, Elger CE, Schramm J: Quantitative and qualitative evaluation of patterns of cerebral language dominance. An amobarbital study. Brain Lang 1994;46:536-564.

4 Oldfield RC: The assessment and analysis of handedness: the Edinburgh inventory. Neuropsychologia 1971;9:97-113.

-5 Stippich C, Rapps N, Dreyhaupt J, Durst A, Kress B, Nennig E, et al: Localizing and lateralizing language in patients with brain tumors: feasibility of routine preoperative functional MR imaging in 81 consecutive patients. Radiology 2007;243:828-836.

-6 Ramsey NF, Sommer IE, Rutten GJ, Kahn RS: Combined analysis of language tasks in fMRI improves assessment of hemispheric dominance for language functions in individual subjects. Neuroimage 2001;13:719-733.

7 Hirsch J, Ruge MI, Kim KH, Correa DD, Victor JD, Relkin NR, et al: An integrated functional magnetic resonance imaging procedure for preoperative mapping of cortical areas associated with tactile, motor, language, and visual functions. Neurosurgery 2000;47:711-721.

-8 Ulmer JL, Hacein-Bey L, Mathews VP, Mueller WM, DeYoe EA, Prost RW, et al: Lesion-induced pseudodominance at functional magnetic resonance imaging: implications for preoperative assessments. Neurosurgery 2004;55:569-579.

9 Wada J, Rasmussen T: Intracarotid injection of sodium amytal for the lateralization of cerebral speech dominance. J Neurosurg 1960;17:226-282.

10 Ojeman G, Ojeman J, Lettich E, Berger M: Cortical language localization in left, dominant hemisphere. An electrical stimulation mapping investigation in 117 patients. J Neurosurg 1989;71:316-326.

11 Amunts K, Schleicher A, Bürgel U, Mohlberg H, Uylings HB, Zilles K: Broca's region revisited: cytoarchitecture and intersubject variability. J Comp Neurol 1999;412:319-341.

12 Schreiber A, Hubbe U, Ziyeh S, Hennig J: The influence of gliomas and nonglial space-occupying lesions on blood-oxygen-level-dependent contrast enhancement. Am J Neuroradiol 2000;21:1055-1063.

13 Nadkarni TN, Andreoli MJ, Nair VA, Yin P, Young BM, Kundu B, et al: Usage of fMRI for pre-surgical planning in brain tumor and vascular lesion patients: task and statistical threshold effects on language lateralization. Neuroimage Clin 2014;7:415-423.

14 Benzagmout M, Gatignol P, Duffau H: Resection of World Health Organization Grade II gliomas involving Broca's area: methodological and functional considerations. Neurosurgery 2007;61:741-752.

15 Plaza M, Gatignol P, Leroy M, Duffau H: Speaking without Broca's area after tumor resection. Neurocase 2009;15:294-310. 


\section{Case Reports in Neurology}
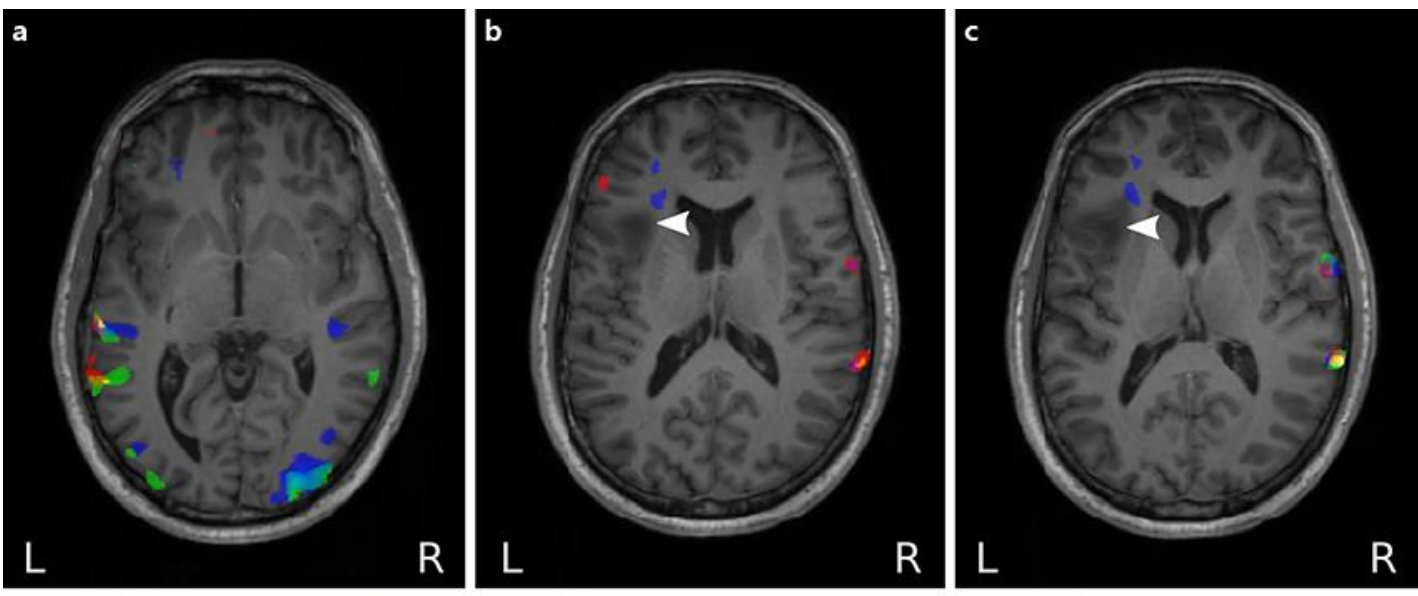

\section{Word generation arun 1 arun 2 Sentence generation arun 1 run 2}

Fig. 1. Activation clusters. a Both runs of both paradigms yielded strong signals in the left dorsal superior temporal gyrus/sulcus, remote from the tumour in the left opercular region and reconcilable with the Wernicke speech area. b, c Corresponding position contralateral: Wernicke homologue. Only the first run of the word generation paradigm suggested activation in the grey matter of the left inferior frontal gyrus, anterior to the tumour (b). b, c A clear activation appeared in the right frontal lobe (first run word generation/both runs sentence generation paradigm), reconcilable with the Broca speech area. Arrowhead indicates tumour. 